\title{
Chemische Eigenschaften von TMP und CTMP, hergestellt aus Kiefernholz und UF-Harz-gebundenen mitteldichten Faserplatten (MDF)
}

\author{
E. Roffael $\cdot$ B. Dix $\cdot$ C. Behn $\cdot$ G. Bär
}

Published online: 20 August 2008

(C) The Author(s) 2008. This article is published with open access at Springerlink.com

\section{Chemical properties of TMP and CTMP prepared from pine wood and UF-bonded medium density fibreboards (MDF)}

Subject Thermo-mechanical (TMP) and chemo-thermomechanical pulps (CTMP) were prepared from pine wood and from UF-bonded MDF made from pine wood. For preparing TMP pine chips as well as UF-bonded MDF were digested under pressure at $170^{\circ} \mathrm{C}$. Thereafter, the digested chips and MDF were defibrated at $170^{\circ} \mathrm{C}$ using a single disc refiner. CTMP was prepared from pine wood with $0.25 \%$ $\mathrm{NaOH}$ (based on dry wood) under the conditions set forth above. CTMP was also made from MDF under two conditions using $0.15 \%$ sodium hydroxide ( $\%$ based on dry MDF) and a mixture of sodium sulphite $(1 \%)$ and sodium hydroxide $(0.25 \%)$. The pulps show distinct differences in their properties: TMP from UF-bonded MDF shows lower extractive content in cold and hot water, lower $\mathrm{pH}$-value and

E. Roffael (匹)

Georg-August-Universität Göttingen,

Büsgenweg 4, 37077 Göttingen, Deutschland

e-mail: eroffae1@gwdg.de

B. Dix

Fraunhofer-Institut für Holzforschung,

Wilhelm-Klauditz-Institut (WKI),

Bienroder Weg 54 E, 38108 Braunschweig, Deutschland

e-mail: brigitte.dix@wki.fraunhofer.de

C. Behn

Büsgen-Institut, Abteilung Molekulare Holzbiotechnologie und Technische Mykologie, Georg-August-Universität Göttingen,

Büsgenweg 2, 37077 Göttingen, Deutschland

G. Bär

Papiertechnische Stiftung (PTS), Institut für Zellstoff und Papier (PTS-IZP),

Pirnaer Str. 37, 01809 Heidenau, Deutschland higher buffering capacity towards alkali of the cold water extractives than the CTMP counterpart. Moreover, CTMP prepared by using sodium hydroxide alone as a pulping agent increases the content of formate and acetate ions in the cold water extractives. The use of a mixture of sodium sulfite and sodium hydroxide as a pulping agent decreases, however, significantly the content of formate and acetate ions in the cold water extractives. This may be due to the buffering action of sodium sulfite. In general, CTMP decreases the formaldehyde release of the fibres, as measured by the flask method. In presence of sodium sulfite as a pulping agent for recycled MDF, the formaldehyde release is slashed to almost $30 \%$ of its original value. The formaldehyde release of CTMP from UF-bonded boards seems to be in the same range as that of TMP from virgin wood.

\section{Material und Methodik}

Kiefernholzhackschnitzel wurden zum einen nach dem TMP-Verfahren bei $170^{\circ} \mathrm{C}$ für $5 \mathrm{~min}$ aufgeschlossen und anschließend in einem Einscheibenrefiner zerfasert. Zum anderen wurden die Hackschnitzel nach dem CTMPVerfahren unter gleichen Bedingungen aufgeschlossen und zerfasert. Bei Anwendung des CTMP-Verfahrens erfolgte der Aufschluss des Holzes in Anwesenheit von 0,25\% Natriumhydroxid (\% Feststoff bezogen auf Trockenmasse Holz). In einem zweiten Versuch wurden UF-Harz-gebundene MDF ebenfalls nach dem thermo-mechanischen (TMP) und chemo-thermo-mechanischen (CTMP) Verfahren bei $170^{\circ} \mathrm{C}$ für $5 \mathrm{~min}$ aufgeschlossen und anschließend in einem Einscheibenrefiner zerfasert. Bei Anwendung des CTMPVerfahrens erfolgte der Aufschluss zum einen in Anwesenheit von $0,15 \%$ Natriumhydroxid (\% Feststoff bezogen auf Trockenmasse MDF) und zum anderen in An- 
Tabelle 1 Chemische Eigenschaften von TMP und CTMP aus Kiefernholz und UF-Harz-gebundenen MDF

Table 1 Chemical properties of TMP and CTMP from pine wood and UF-bonded MDF

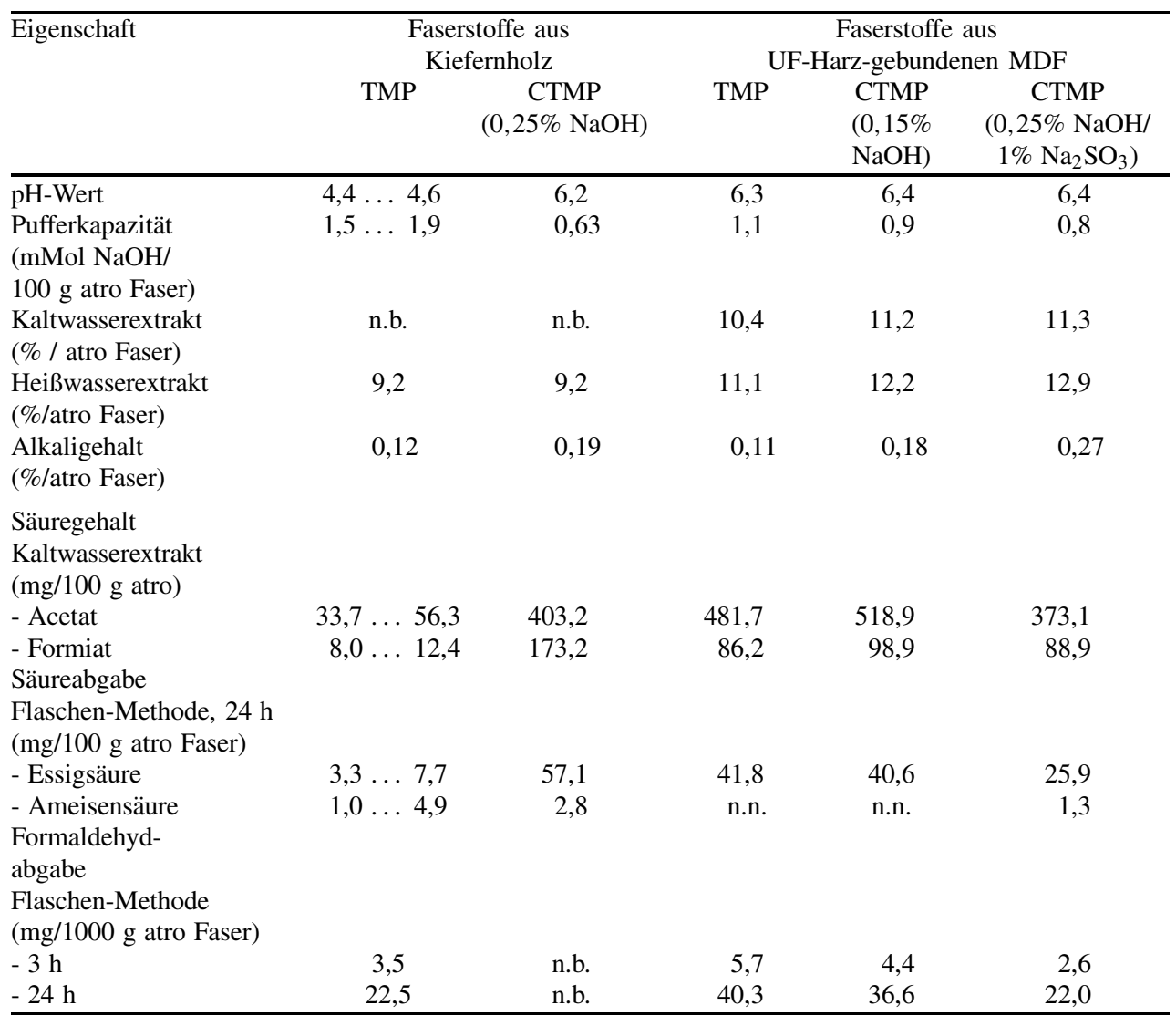

atro: absolut trocken; n.n.: nicht nachgewiesen; n.b.: nicht bestimmt wesenheit einer Mischung aus 0,25\% Natriumhydroxid und 1\% Natriumsulfit (\% Feststoff bezogen auf Trockenmasse MDF). An den hergestellten Fasern wurden der pH-Wert der Kaltwasserextrakte, der Gehalt an in Kaltund Heißwasser löslichen Bestandteilen, die Pufferkapazität der Kaltwasserextrakte gegenüber Alkali, der Gehalt an Acetat- und Formiationen in den Kaltwasserextrakten und die Abgabe an flüchtigen Säuren in Anlehnung an das Prinzip der Flaschenmethode (EN 717-3 1996) bestimmt. Ferner wurde für die hergestellten Faserstoffe die Formaldehydabgabe nach der Flaschenmethode (EN 7173 1996) ermittelt. Die Ergebnisse sind in der Tabelle 1 zusammengestellt.

\section{Ergebnisse und Diskussion}

Die Ergebnisse (Tabelle 1) zeigen, dass der Gehalt an Formiat- und Acetationen in den wässrigen Extrakten der ohne Zugabe von Chemikalien thermo-mechanisch aufgeschlossenen MDF um das Zehnfache höher liegt als in den wässrigen Extrakten des thermo-mechanisch aufgeschlossenen Holzes. Die Ergebnisse lassen ferner deutlich werden, dass auch die Abgabe an Essigsäure aus den ohne Chemika- lien aufgeschlossenen MDF hergestellten Fasern wesentlich höher ist als die aus den entsprechenden Fasern, die direkt aus dem Holz stammen. Dieses Ergebnis steht womöglich damit in Zusammenhang, dass während des Aufschlusses das Bindemittel in UF-gebundenen MDF unter Entstehung von Ammoniak abgebaut wird und hierdurch der Aufschluss chemo-thermo-mechanisch erfolgt (vgl. hierzu Roffael et al. 2008). Das Ammoniak erhöht den pH-Wert der Fasern und führt zum stärkeren Abbau der Acetylgruppen im Holz als im Falle des Aufschlusses von bindemittelfreien Hackschnitzeln.

Im Vergleich zum Aufschluss der UF-Harz-gebundenen MDF nach dem TMP-Verfahren erhöht der Aufschluss der MDF nach dem CTMP-Verfahren geringfügig den $\mathrm{pH}-$ Wert und verringert deutlich die alkalische Pufferkapazität der Kaltwasserextrakte. Der Gehalt an Kalt- und Heißwasserextraktstoffen nimmt beim Aufschluss nach dem CTMP-Verfahren zu. Der Gehalt an flüchtigen Säuren in den Kaltwasserextrakten steigt beim Aufschluss nach dem CTMP-Verfahren mit Natriumhydroxid allein deutlich an. Bei Verwendung von einem Gemisch aus Natriumhydroxid und Natriumsulfit als Aufschlusschemikalie wird der Gehalt an Formiat- und Acetationen in den Kaltwasserextrakten deutlich verringert. Dies ist - trotz des höheren Anteils an 
Natriumhydroxid beim Aufschluss - vermutlich auf die abpuffernde Wirkung von Natriumsulfit zurückzuführen, die bekanntlich den Abbau an Kohlenhydraten - als ,,precursor“ für die Bildung von flüchtigen Säuren - verringert. Interessant ist ferner die Feststellung, dass die Formaldehydabgabe der nach dem CTMP-Verfahren aus MDF hergestellten Fasern generell niedriger ist als die der nach dem TMP-Verfahren gefertigten. Besonders gering ist die Formaldehydabgabe der Fasern, die in Anwesenheit von Natriumhydroxid und Natriumsulfit hergestellt wurden; die Formaldehydabgabe der CTMP liegt hier um ca. 50\% niedriger als die der TMP. Insofern werden die an Holzfasern getroffenen Feststellungen (Roffael et al. 1994a, Roffael et al. 1994b, Roffael et al. 1995) auch an Fasern aus Recycling-MDF bestätigt. Interessanterweise sind keine großen Unterschiede zwischen der Formaldehydabgabe der TMP aus Kiefernholz und der CTMP $(0,25 \%$ $\mathrm{NaOH}+1 \% \mathrm{Na}_{2} \mathrm{SO}_{3}$ ) aus MDF - hergestellt aus Kiefernholz - festzustellen.

Förderung: Das Verbundvorhaben zwischen der Universität Göttingen, dem Fraunhofer-Institut für Holzforschung und dem Institut für Zellstoff und Papier der Papiertechnischen Stiftung wurde mit Mitteln des Bundesministeriums für Wirtschaft und Technologie (BMWi) durch die Arbeitsgemeinschaft industrieller Forschungsvereinigungen (AiFNr. 14801 BG) über den Internationalen Verein für Technische Holzfragen (iVTH) gefördert.
Open Access Dieser Artikel wird zu den Bedingungen der "Creative Commons Attribution Noncommercial License" zur Verfügung gestellt. Damit ist eine nichtkommerzielle Nutzung, Verbreitung und Vervielfältigung erlaubt, sofern die Autoren des Artikels und die genaue Quelle angegeben sind.

\section{Literatur}

EN 717-3 (1996) Holzwerkstoffe - Bestimmung der Formaldehydabgabe. Teil 3: Formaldehydabgabe nach der Flaschen-Methode

Roffael E, Dix B, Bär G, Bayer R (1994a) Über die Eignung von thermo-mechanischem und chemo-thermo-mechanischem Holzstoff (TMP und CTMP) aus Buchen- und Kiefernholz für die Herstellung von mitteldichten Faserplatten (MDF). Teil 1: Aufschluss des Holzes und Eigenschaften der Faserstoffe. Holz RohWerkst 52:239-246

Roffael E, Dix B, Bär G, Bayer R (1994b): Über die Eignung von thermo-mechanischem und chemo-thermo-mechanischem Holzstoff (TMP und CTMP) aus Buchen- und Kiefernholz für die Herstellung von mitteldichten Faserplatten (MDF). Teil 2: Eigenschaften von MDF aus Buchen-Faserstoff. Holz Roh- Werkst 52:293-298

Roffael E, Dix B, Bär G, Bayer R (1995): Über die Eignung von thermo-mechanischem und chemo-thermo-mechanischem Holzstoff (TMP und CTMP) aus Buchen- und Kiefernholz für die Herstellung von mitteldichten Faserplatten (MDF). Teil 3: Eigenschaften von aus Kiefern-Faserstoff hergestellten MDF. Holz Roh- Werkst 53:8-11

Roffael E, Dix B, Behn C, Bär G (2008): Benetzung von TMP und CTMP aus Holz sowie gebrauchten mitteldichten Faserplatten (MDF). Holz Roh- Werkst, doi: 10.1007/s00107-008-0265-2 